\title{
Three-Dimensional Echocardiography of Left Atrial Appendage Thrombus
}

\author{
Gazala N. Khan, M.B.B.S., Ismail T. Dairywala, M.D., Zheng Liu, M.D., Peng Li, M.D., \\ Jim Carroll, M.D., and Mani A. Vannan, M.B.B.S. \\ The Division of Cardiology, Department of Internal Medicine, University of Michigan Health \\ System, Ann Arbor, Michigan
}

Transesophageal echocardiography (TEE) is superior to transthoracic echocardiography (TTE) in evaluating the left atrial appendage (LAA) as a potential cardiac source of embolus in patients with stroke. We describe two such patients in whom a TEE and subsequently a three-dimensional (3-D) reconstruction of the LAA were performed. These case reports show that 3-D echocardiography provides better visualization of LAA anatomy, and that a 3-D description of LAA morphology may be the basis of describing normal and abnormal LAA. (ECHOCARDIOGRAPHY, Volume 18, February 2001)

\section{Case Report 1}

A 76-year-old male patient with a history of nonvalvular atrial fibrillation and hypertension presented with an acute cerebrovascular accident with left-sided hemiparesis and slurred speech. Computed tomography scan of his brain showed a maturing subacute right cerebral infarction. Carotid Duplex showed abnormal velocities in the right internal carotid artery suggestive of distal occlusion. A two-dimensional (2-D) transesophageal echocardiography (TEE) was performed, revealing a left atrial appendage (LAA) thrombus (Fig. 1A and 1B) and spontaneous contrast in the left atrium. Three-dimensional echocardiographic reconstruction revealed the thrombus (Fig. 1C, black arrow) in the LAA from a "zoomed" frontal view along with the ascending aorta (AO). The thrombus (Fig. 1D, double black arrows) also can be seen when viewed from the roof of the left atrium (LA). The stalk (Fig. 1E, single black arrow) attaching the thrombus to the atrial wall is clearly visualized. The mitral valve also is shown during diastole (Fig. 1D) and systole (Fig. 1E).

\section{Case Report 2}

A 71-year-old female with a history of mitral regurgitation presented with atrial fibrillation.

Address for correspondence and reprint requests: Mani A. Vannan, M.B.B.S., University of Michigan Health System, Women's L3119, 1500 E. Medical Center Drive, Ann Arbor, MI 48109. Fax: 734-763-7390; E-mail: mvannan@umich.edu
A 2-D TEE revealed moderate mitral regurgitation, a patent foramen ovale, and chamber enlargement confined to the atria. It also revealed spontaneous echo contrast in the LAA combined with sludge (Fig. $2 \mathrm{~A}$ and $2 \mathrm{~B}$, white arrow in B pointing to the sludge). Three-dimensional reconstruction (Fig. 2C, 2D, and 2E) shows bilobed LAA in different orientation. The sludge is clearly visualized in Figures 2D and $2 \mathrm{E}$ due to its different echo density (black arrows pointing to the sludge). Figure $2 \mathrm{E}$ is an "en face" view of the LAA, which is unique to 3-D echocardiography.

\section{Discussion}

Cardiogenic embolism accounts for $\geq 15 \%$ of ischemic strokes. ${ }^{1}$ Of these, the LAA clot is an important source. With the advent of TEE, tremendous insight has been gained in visualizing thrombi of the LAA, delineating its structure and function, and in determining potential high-risk states that could lead to thromboembolic sequelae. ${ }^{2,3}$

TEE has been shown to be superior to TTE in the assessment of LAA structure and function. In a study conducted by Pearson et al. ${ }^{4}$ in a population that was comprised of patients with unexplained stroke or transient ischemic attack, TEE identified a potential cardiac source of embolus in 57\% of the overall study group compared with $15 \%$ by TTE $(\mathrm{P}<0.0005)$. Similar observations documenting the superiority of TEE over TTE in visualizing LAA thrombi and elucidating its function have been made in several studies. ${ }^{2-5}$

In studies of high-risk patients who are TEE 

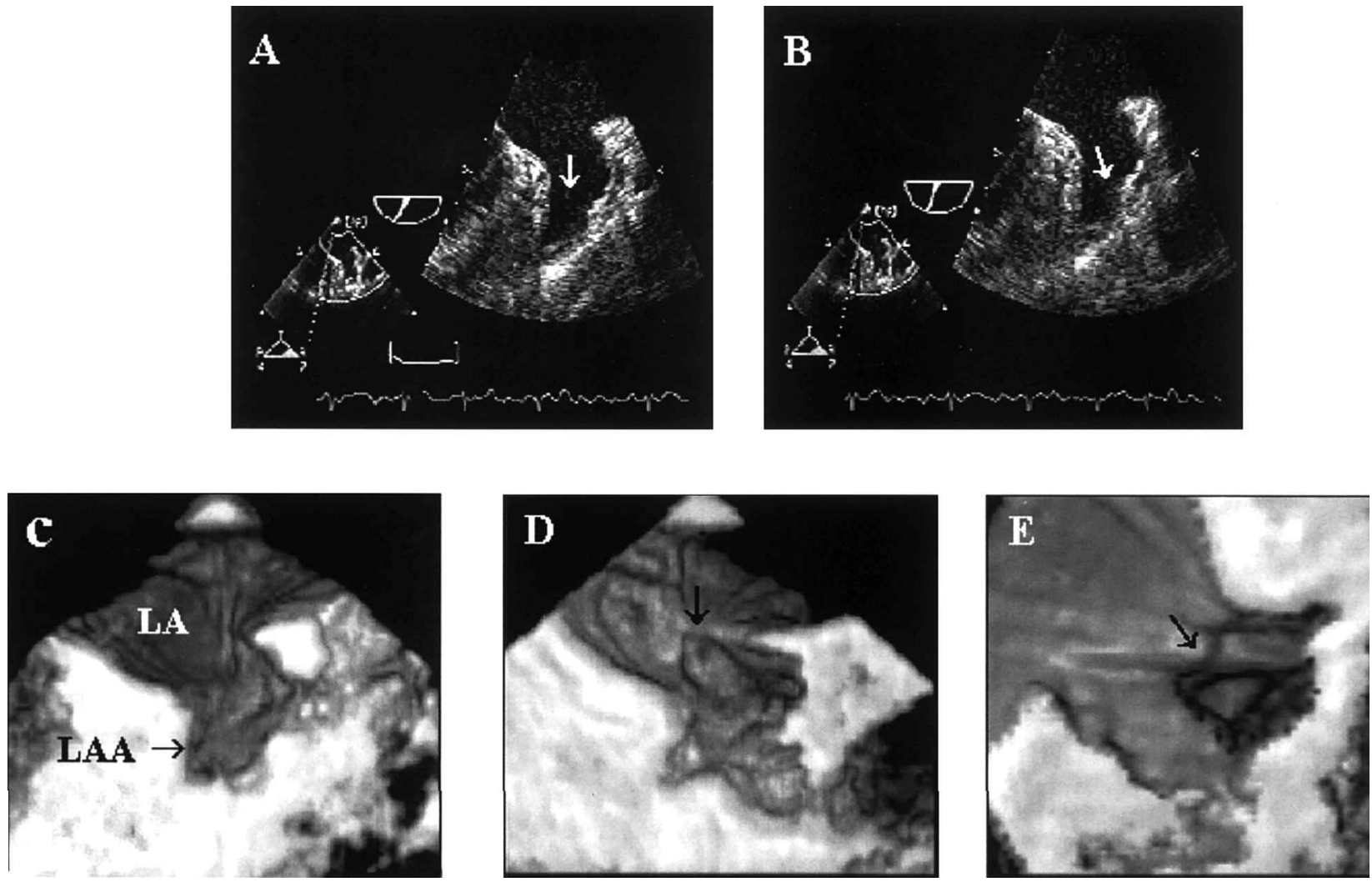

Figure 1. A. 2-D TEE image of the left atrial appendage (LAA-white arrow). B. 2-D image of the LAA showing spontaneous contrast and sludge (white arrow). C. 3-D reconstructed image of the LAA. D. 3-D reconstructed image showing sludge (black arrow) in the LAA. E. 3-D reconstructed "en face" view of the LAA showing the presence of sludge (black arrows).

negative for LAA thrombi, LAA anatomy (bilobed vs multilobed), LAA flow velocity (lowflow states predispose to thrombus formation), and LAA dimensions (width, length, and crosssectional area), occurrence of Spontaneous Echo Contrast (SEC) indicating low-flow states has been shown to be determinants of thromboembolic sequelae. ${ }^{6}$ A prototype study would be the recent ELAT study conducted by Stollberger et al. ${ }^{7}$ in patients with atrial fibrillation, in whom a univariate analysis was done between the echocardiographic characteristics of the LAA and the occurrence of thromboembolic events. The results were as follows: thrombi $(\mathrm{P}=0.009)$; length of the LAA $(\mathrm{P}=0.03)$; and width of the LAA $(\mathrm{P}=0.01){ }^{7}$ In the study done by Beppu et al., the incidence of thrombus formation in patients with well-marked SEC was $60 \%$, as opposed to $9 \%$ in patients without welldefined SEC. ${ }^{8}$ Sludge is a frequent component of SEC.

However, TEE has two main limitations. First, measurement of LAA cross-sectional area is subject to considerable interobserver variability ${ }^{9,10}$ owing to its complex 3 -D orientation, which limits the determination of standard tomographic imaging planes. Second, exclusion by TEE of preexisting atrial thrombi before cardioversion does not preclude the formation of atrial thrombi after cardioversion. It has been hypothesized that atrial stasis could contribute to this or that it could be due to a decreased sensitivity of TEE for sludge. ${ }^{11,12}$

Three-dimensional echocardiographic reconstruction enables excellent visualization of LAA anatomy, dimensions, and function. Further studies that provide 3-D quantification of LAA morphology may be the basis of characterizing the LAA in normal and abnormal states. Our case studies show that 3-D echocardiography has sufficient resolution to not only show large thrombi (Case Study 1), but even significantly smaller sludge (Case Study 2). Thus, 3-D imaging of the LAA may provide valuable anatomical and physiologi- 

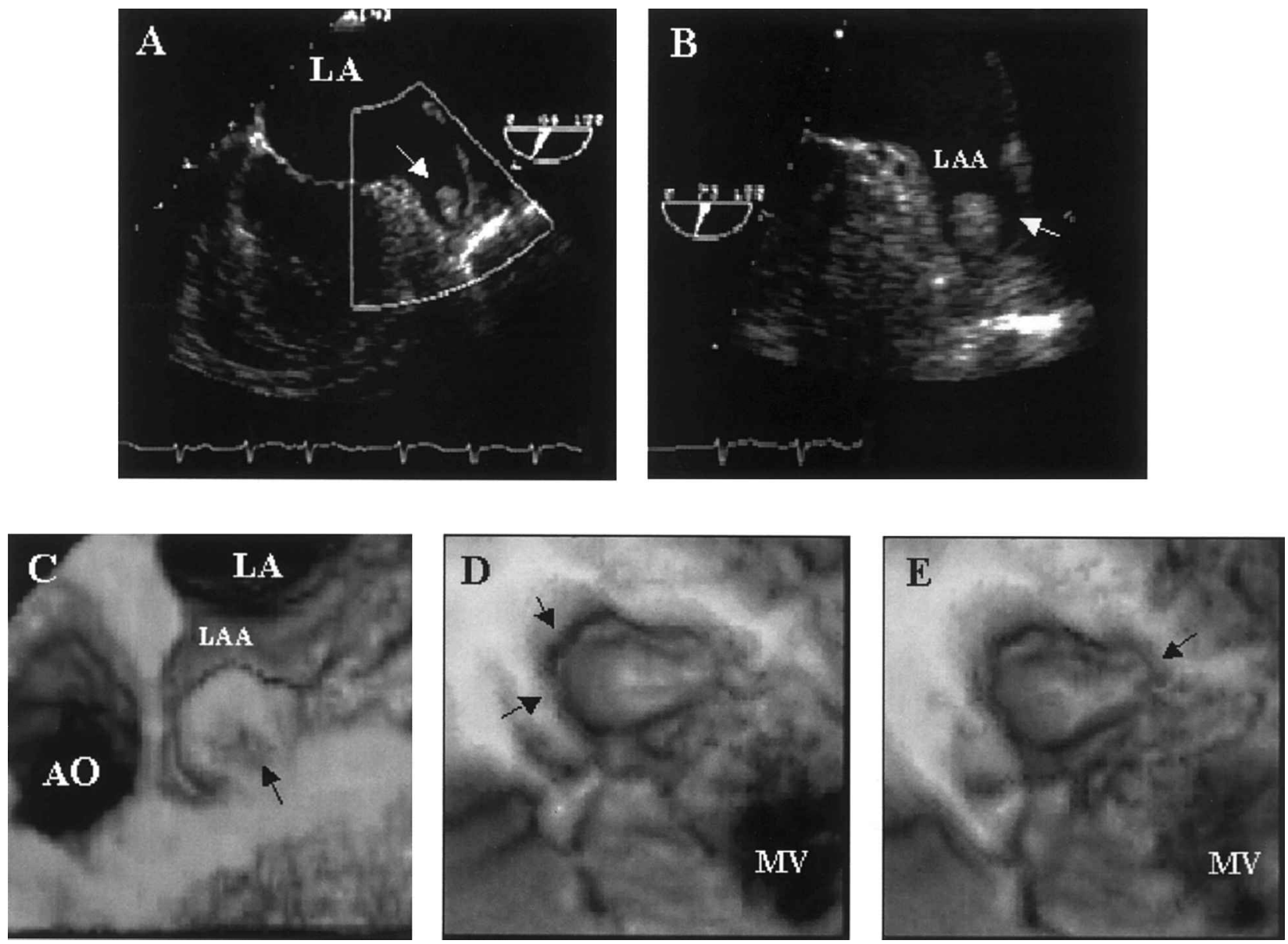

Figure 2. A and B 2-D TEE image of a thrombus (white arrow) in the LAA. C. 3-D reconstructed image of the thrombus (black arrow) in the LAA. Also visualized are the left atrium (LA) and the ascending aorta (AO). D. 3-D reconstructed image of the thrombus (black arrows) as seen from the roof of the LA. The mitral valve (MV) during diastole is also seen. E. A 3-D reconstructed image showing the stalk (black arrow) of the thrombus and the MV during systole.

cal data in evaluation of patients for cardiac source of embolus. Currently, one of the limitations of 3-D echocardiography is that it is a time-intensive technique. Also, dense spontaneous echo contrast may cause problems with 3 -D reconstruction, although this did not interfere with image reconstruction in the cases reported here.

\section{References}

1. Cerebral Embolism Task Force: Cardiogenic brain embolism. Arch Neurol 1986;43:71-84.

2. Omran H, Jung W, Rabahieh R, et al: Imaging of thrombi and assessment of left atrial appendage function: A prospective study comparing transthoracic and transesophageal echocardiography. Heart 1999; 81:192-198.

3. Lee RJ, Bartzokis T, Yeoh TK, et al: Enhanced detection of intracardiac sources of cerebral emboli by transesophageal echocardiography. Stroke 1991;22: 734-739.

4. Pearson AC, Labovitz AJ, Tatineni S, et al: Superiority of transesophageal echocardiography in detecting cardiac source of embolism in patients with cerebral ischemia of uncertain etiology. $\mathrm{J}$ Am Coll Cardiol 1991;7:66-72.

5. Cujec B, Polasek P, Voll C, et al: Transesophageal echocardiography in the detection of potential cardiac source of embolism in stroke patients. Stroke 1991;22: 727-733.

6. Asinger RW, Koehler J, Pearce LA, et al: Pathophysiologic correlates of thromboembolism in nonvalvular atrial fibrillation: II. Dense spontaneous echocardiographic contrast (The Stroke Prevention in Atrial Fibrillation [SPAF-III] study). J Am Soc Echocardiogr 1999;12:1088-1096.

7. Stollberger C, Chnupa P, Kronik G, et al: Transesophageal echocardiography to assess embolic risk in patients with atrial fibrillation. ELAT study group. Embolism in left atrial thrombi. Annals of Int Med 1998; 128:630-638. 
KHAN, ET AL.

8. Beppu S: Hypercoagulability in the left atrium: Part I: Echocardiography. J Heart Valve Dis 1993; 2:18-24.

9. Pollick C, Taylor D: Assessment of left atrial appendage function by transesophageal echocardiography: Implications for the development of thrombus. Circulation 1991;84:223-231.

10. Ito T, et al: Influence of left atrial function on Doppler transmitral and pulmonary venous flow patterns in dilated and hypertrophic cardiomyopathy: Evaluation of left atrial appendage function by transesophageal echocardiography. (Abstract) Am Heart J 1996;131: $122-130$.

11. Black IW, Fatkin D, Sagar KB, et al: Exclusion of atrial thrombus by TEE does not preclude embolism after cardioversion of atrial fibrillation: A multicenter study. Circulation 1994;89:2509-2513.

12. Agmon Y, Khanderia BK, Gentile F, et al: Echocardiographic assessment of the left atrial appendage. J Am Coll Cardiol 1999;34:1867-1877. 